\title{
SOME RATE INVARIANT SEQUENCE TRANSFORMATIONS
}

\author{
DAVID F. DAWSON
}

The basis for comparing convergence rates of sequences used here is as follows: If $\left\{x_{p}\right\}$ converges to $X$ and $\left\{y_{p}\right\}$ converges to $Y$, then " $\left\{x_{p}\right\}$ converges faster than $\left\{y_{p}\right\}$ " means that $\lim _{n \rightarrow \infty}\left(x_{n}-X\right) /\left(y_{n}-Y\right)$ $=0$. Similarly, if $\sum a_{p}$ and $\sum b_{p}$ are convergent complex series, then " $\sum a_{p}$ converges faster than $\sum b_{p}$ " means that

$$
\lim _{n \rightarrow \infty} \sum_{p=n}^{\infty} a_{p} / \sum_{p=n}^{\infty} b_{p}=0
$$

[3]. "A sequence-to-sequence transformation $A$ is rate (of convergence) invariant" means that if each of $x$ and $A x$ is a convergent sequence, then neither sequence converges faster than the other. If $\left\{x_{p}\right\}$ and $\left\{y_{p}\right\}$ diverge to $\infty$, then " $\left\{y_{p}\right\}$ diverges faster than $\left\{x_{p}\right\} "$ means that $\lim _{n \rightarrow \infty} x_{n} / y_{n}=0$.

In this note, certain matrices, regarded here as sequence-to-sequence transformations, which are convergence invariant (sum only convergent sequences) are given and shown to be rate invariant. In general, a convergence invariant matrix need not be rate invariant, as shown by the following example. Suppose $\left\{x_{p}\right\}$ converges to 1 , $x_{p} \neq 1, x_{p} \neq 0, p=1,2,3, \cdots$. Let $A$ be the diagonal matrix such that $a_{i i}=x_{i}$, and let $x^{\prime}=\left\{1 / x_{p}\right\}$. Then $A x^{\prime}$ converges faster than $x^{\prime}$, while $y=\{1,1,1, \cdots\}$ converges faster than $A y$. Thus the convergence invariant matrix $A$ speeds up convergence in one case and slows it down in another.

As a corollary to our study, we have the result (Theorem 3 ) that if $\left\{p_{i}\right\}$ is a complex sequence such that $\sum a_{i}$ and $\sum p_{i} a_{i}$ converge or diverge together for all choices of $\sum a_{i}$, then for each series $\sum b_{i}$ which is convergent, neither of the series $\sum b_{i}$ and $\sum p_{i} b_{i}$ converges faster than the other, i.e., the series $\sum b_{i}$ and $\sum p_{i} b_{i}$ are rate invariant.

THEOREM 1. If $\sum\left|a_{p}\right|$ converges and $d_{p} \rightarrow k$, then the convergence preserving matrix $A=\left(a_{p q}\right)$, defined by $a_{p q}=0$ if $q>p, a_{p q}=d_{p}$ if $p=q$, $a_{p q}=a_{q}$ if $q<p$, speeds up the convergence of some null sequence if and only if $k=0$.

Proof. Suppose $k \neq 0$, but there exists a complex sequence $x=\left\{x_{p}\right\}$

Received by the editors March 6, 1963 and, in revised form, May 5, 1963. 
convergent to 0 such that $A x$ converges faster than $x$. Let $A x=\left\{y_{p}\right\}$ and note that $\lim _{n \rightarrow \infty} y_{n}=\sum_{p=1}^{\infty} a_{p} x_{p}$. Let $\lim _{n \rightarrow \infty} y_{n}=Y$. Thus our assumption is that $\lim _{i \rightarrow \infty}\left(y_{i}-Y\right) /\left(x_{i}-0\right)=0$, which means that $x$ contains at most a finite number of zero terms. Let $N$ be a positive integer such that if $q>N$, then $x_{q} \neq 0$. Thus, if $n>N$, then

$$
\frac{y_{n}-Y}{x_{n}-0}=d_{n}-\frac{a_{n} x_{n}+a_{n+1} x_{n+1}+\cdots}{x_{n}} .
$$

We note that for infinitely many values of $i,\left|x_{i}\right| \geqq\left|x_{i+p}\right|$, $p=1,2,3, \cdots$, since $x$ converges to 0 . Hence, since $\sum\left|a_{p}\right|$ converges,

$$
\liminf _{i \rightarrow \infty}\left|\frac{a_{i} x_{i}+a_{i+1} x_{i+1}+\cdots}{x_{i}}\right|=0,
$$

and therefore, since our assumption, in light of (1), implies that $\lim _{p \rightarrow \infty}\left(a_{p} x_{p}+a_{p+1} x_{p+1}+\cdots\right) / x_{p}$ exists, we have

$$
\lim _{i \rightarrow \infty} \frac{a_{i} x_{i}+a_{i+1} x_{i+1}+\cdots}{x_{i}}=0 .
$$

Thus from (1) and the fact that $d_{p} \rightarrow k$, we see that

$$
\lim _{i \rightarrow \infty}\left(y_{i}-Y\right) /\left(x_{i}-0\right)=k \neq 0 .
$$

But this is a contradiction. Hence if $k \neq 0$, there exists no complex null sequence $x$ such that $A x$ converges faster than $x$.

On the other hand, if $k=0$, it is easily seen from (1) that any null sequence $x=\left\{x_{p}\right\}$ such that for each positive integer $i,\left|x_{i}\right|>\left|x_{i+p}\right|$, $p=1,2,3, \cdots$, has the property that $A x$ converges faster than $x$. This completes the proof of the theorem.

We now add a condition to the hypothesis of Theorem 1 which gives a kind of "translation" property and makes the conclusion valid for convergent sequences which need not be null.

THEOREM 2. If the complex sequence $\left\{d_{p}\right\}$ is of bounded variation, $k$ being its limit, and $a_{p}=d_{p}-d_{p+1}, p=1,2,3, \cdots$, then the matrix $A$, as defined in the statement of Theorem 1, speeds up the convergence of some sequence if and only if $k=0$.

Proof. Suppose $k \neq 0$, but there exists $x=\left\{x_{p}\right\}$ convergent to $X$ such that $A x$ converges faster than $x$. Let $N$ be a positive integer such that if $n>N$, then $x_{n} \neq X$. Denote $A x$ by $\left\{y_{p}\right\}$ and let $\lim _{n \rightarrow \infty} y_{n}$ 
$=Y$. Our assumption is that $\lim _{n \rightarrow \infty}\left(y_{n}-Y\right) /\left(x_{n}-X\right)=0$. If $n>N$, then

$$
\begin{aligned}
y_{n}-Y & =d_{n} x_{n}-k X-\sum_{i=n}^{\infty} a_{i} x_{i} \\
& =d_{n} x_{n}-X \sum_{i=n}^{\infty} a_{i}+\sum_{i=n}^{\infty} a_{i}\left(X-x_{i}\right)-k X \\
& =d_{n} x_{n}-d_{n} X+k X+\sum_{i=n}^{\infty} a_{i}\left(X-x_{i}\right)-k X,
\end{aligned}
$$

and so

$$
\frac{y_{n}-Y}{x_{n}-X}=d_{n}+\frac{a_{n}\left(X-x_{n}\right)+a_{n+1}\left(X-x_{n+1}\right)+\cdots}{x_{n}-X} .
$$

As in the proof of Theorem 1,

$$
\liminf _{i \rightarrow \infty}\left|\frac{a_{i}\left(X-x_{i}\right)+a_{i+1}\left(X-x_{i+1}\right)+\cdots}{x_{i}-X}\right|=0,
$$

and so $\lim _{i \rightarrow \infty}\left(y_{i}-Y\right) /\left(x_{i}-X\right)=k \neq 0$, which contradicts the assumption that $A x$ converges faster than $x$.

If $k=0$, then, as in the proof of Theorem 1 , any null sequence $x=\left\{x_{p}-X\right\}$ such that for each positive integer $i,\left|x_{i}-X\right|$ $>\left|x_{i+p}-X\right|, p=1,2,3, \cdots$, has the property that $A x$ converges faster than $x$. This completes the proof of the theorem.

THEOREM 3. If $\sum_{i=N}^{\infty}\left|1-p_{i+1} / p_{i}\right|$ converges for some $N$, then for each convergent series $\sum a_{i}$, the series $\sum a_{i}$ and $\sum p_{i} a_{i}$ are rate invariant.

Proof. Suppose no $p_{i}=0$. Let $H=\left(h_{i j}\right)$ and $H^{\prime}=\left(h_{i j}^{\prime}\right)$ be matrices defined as follows $[1]$ :

$$
h_{i j}=\left\{\begin{array}{l}
0 \text { if } i<j, \\
p_{i} \text { if } i=j, \\
p_{j}-p_{j+1} \text { if } j<i,
\end{array} \quad h_{i j}^{\prime}=\left\{\begin{array}{l}
0 \text { if } i<j, \\
1 / p_{i} \text { if } i=j, \\
1 / p_{j}-1 / p_{j+1} \text { if } j<i .
\end{array}\right.\right.
$$

It is easily verified that if $\sum a_{p}$ is a complex series, then $H$ transforms the sequence $\left\{a_{1}+a_{2}+\cdots+a_{i}\right\}_{i=1}^{\infty}$ into the sequence $\left\{p_{1} a_{1}+p_{2} a_{2}+\cdots+p_{i} a_{i}\right\}_{i=1}^{\infty}$ and $H \cdot H^{\prime}=H^{\prime} \cdot H=I$ (the identity matrix). Since $\sum\left|1-p_{i+1} / p_{i}\right|$ converges, $\left\{p_{i}\right\}$ converges to a complex number $k \neq 0$ and each of the series $\sum\left|p_{i+1}-p_{i}\right|$ and 
$\sum\left|1 / p_{i+1}-1 / p_{i}\right|$ converges [2]. Hence $H$ and $H^{\prime}$ both have the properties attributed to the matrix $A$ of Theorem 2, and the principal diagonals converge, respectively, to $k \neq 0$ and $1 / k$. Thus by Theorem 2, for each convergent series $\sum a_{i}$, neither of the series $\sum a_{i}$ and $\sum p_{i} a_{i}$ converges faster than the other. In case some $p_{i}=0$, we consider $\left\{p_{i}\right\}_{i=N}^{\infty}$ and the series $\sum_{i=N}^{\infty} a_{i}$ and $\sum_{i=N}^{\infty} p_{i} a_{i}$, where $p_{i} \neq 0$ for $i \geqq N$, and the proof is similar to the above.

As partial converses of Theorem 3, we have the two theorems which follow.

THEOREM 4. If $\left\{p_{i}\right\}$ is such that $H$ is convergence preserving and for each convergent series $\sum a_{i}$, the series $\sum a_{i}$ and $\sum p_{i} a_{i}$ are rate invariant, then $\sum_{i=N}^{\infty}\left|1-p_{i+1} / p_{i}\right|$ converges for some $N$.

Proof. Consider first the case that no $p_{i}=0$. Suppose $H^{\prime}$ is not convergence preserving. This means that $\sum_{i=1}^{\infty}\left|p_{i+1}-p_{i}\right|$ converges and $\left\{p_{i}\right\}$ converges to 0 . Hence by Theorem 2 there exists a sequence $x$ such that $H x$ converges faster than $x$. Thus for the hypothesis to hold, both $H$ and $H^{\prime}$ must be convergence preserving, and so $\sum_{i=1}^{\infty}\left|1-p_{i+1} / p_{i}\right|$ converges, and the proof is complete for the case we considered. In case some $p_{i}=0$, the proof is similar, since there exists $N$ such that $p_{i} \neq 0$ for $i \geqq N$.

THEOREM 5. If $\left\{p_{i}\right\}$ is such that $H^{\prime}$ is convergence preserving and for each convergent series $\sum p_{i} a_{i}$, the series $\sum a_{i}$ and $\sum p_{i} a_{i}$ are rate invariant, then $\sum_{i=1}^{\infty}\left|1-p_{i+1} / p_{i}\right|$ converges.

Proof. Suppose $H$ is not convergence preserving. Then $\left|p_{i}\right| \rightarrow \infty$ as $i \rightarrow \infty$. Hence by Theorem 2 , there exists a convergent sequence $x$ such that $H^{\prime} x$ converges faster than $x$. But this is a contradiction, and so $H$ is convergence preserving. Thus $\sum_{i=1}^{\infty}\left|1-p_{i+1} / p_{i}\right|$ converges, and the proof is complete.

The remainder of the paper is devoted to theorems which involve divergence, but which are analogous to some of the above theorems. The proof of Theorem 6 will be given, but the proof of Theorem 7 will be omitted because of the similarity with Theorem 3 .

TheoRem 6. If $\sum\left|a_{p}\right|$ converges and $d_{p} \rightarrow k \neq 0$, then the matrix $A$, as defined in Theorem 1, has the following properties:

(a) there exists a sequence $x$ such that both $x$ and Ax diverge to $\infty$, and

(b) if both $x$ and $A x$ diverge to $\infty$, then $x$ does not diverge faster than $A x$.

Proof. We will prove (b) first. Suppose $x=\left\{x_{p}\right\}$ and $A x$ both diverge to $\infty$ and $x$ diverges faster than $A x$. Let $A x=\left\{c_{p}\right\}$. Then 
$\lim _{n \rightarrow \infty} c_{n} / x_{n}=0$. Let $N_{1}$ be a positive integer such that $x_{i} \neq 0$ if $i>N_{1}$. Let $N_{2}>1$ be an integer such that $\sum_{p=N_{2}}^{\infty}\left|a_{p}\right|<|k| / 2$. Let $N>N_{1}+N_{2}$. Then if $n>N$, we have

$$
\begin{aligned}
\frac{c_{n}}{x_{n}} & =\frac{a_{1} x_{1}+a_{2} x_{2}+\cdots+a_{n-1} x_{n-1}+d_{n} x_{n}}{x_{n}} \\
& =d_{n}+\frac{a_{1} x_{1}+\cdots+a_{N_{2-1}} x_{N_{2}-1}}{x_{n}}+\frac{a_{N_{2}} x_{N_{2}}+\cdots+a_{n-1} x_{n-1}}{x_{n}} .
\end{aligned}
$$

We note that for infinitely many values of $i,\left|x_{i}\right| \geqq\left|x_{i-j}\right|, j=1,2$, $\cdots, i-1$, since $x$ diverges to $\infty$. Thus

$$
\liminf _{i \rightarrow \infty}\left|\frac{a_{N_{2}} x_{N_{2}}+\cdots+a_{i-1} x_{i-1}}{x_{i}}\right|<\left|\frac{k}{2}\right| .
$$

Thus from our assumption that $\lim _{n \rightarrow \infty} c_{n} / x_{n}$ exists, we see from (2) that $\lim _{i \rightarrow \infty}\left|c_{i} / x_{i}\right|>|k| / 2>0$. But this is a contradiction of the assumption that $x$ diverges faster than $A x$.

To prove (a), we let $x=\left\{x_{p}\right\}$ be any sequence which diverges to $\infty$ such that $\left|x_{n}\right|<\left|x_{n+1}\right|, n=1,2,3, \cdots$. Let $A x=\left\{y_{p}\right\}$. Then from (2) we see that $\lim \inf _{i \rightarrow \infty}\left|y_{i} / x_{i}\right|>|k| / 2$. Hence $\left\{y_{p}\right\}$ diverges to $\infty$.

THEOREM 7. If $\sum_{i=N}^{\infty}\left|1-p_{i+1} / p_{i}\right|$ converges for some $N$ and $\sum a_{i}$ and $\sum p_{i} a_{i}$ diverge to $\infty$, then neither series diverges faster than the other.

\section{REFERENCES}

1. D. F. Dawson, Concerning a theorem of Hadamard, Amer. Math. Monthly 69 (1962), 981-983.

2. - Convergence of continued fractions of Stieltjes type, Proc. Amer. Math. Soc. 10 (1959), 12-17.

3. Konrad Knopp, Infinite sequences and series, Dover, New York, 1956.

North TeXas State University 